\title{
Low-dose ketamine as an adjuvant for pain control in a cancer patient: a case report
}

\author{
Sapan Patel ${ }^{1}$, Vivas Tatachar $^{1}$, Aditya Bikram Singh ${ }^{2}$, Julia Galea $^{3}$, Emma Fattakhov $^{4}$, Gurjinder Kaur ${ }^{1}$ \\ ${ }^{1}$ Department of Physiology, Touro College of Osteopathic Medicine, Middletown, NY, USA; ${ }^{2}$ Rutgers New Jersey Medical School, Newark, NJ, \\ USA; ${ }^{3}$ Pharmacy Department, Garnet Health Medical Center, Middletown, NY, USA; ${ }^{4}$ Palliative Care Medicine, Garnet Health Medical Center, \\ Middletown, NY, USA \\ Correspondence to: Gurjinder Kaur. Department of Physiology, Touro College of Osteopathic Medicine, Middletown, NY, USA. \\ Email: gurjinder.kaur14@touro.edu.
}

\begin{abstract}
Cancer patients often suffer from pain related problems such as under-treatment of pain, ineffective and persistent opioid administration as well as adverse opioid use outcomes. There is a growing need for non-opioid analgesic alternatives for patients undergoing treatment for obstinate pain. Ketamine is a fast-acting $\mathrm{N}$-methyl-D-aspartate (NMDA) receptor antagonist that has been emerging as an effective medication for pain alleviation. While protocols have been established for the use of Low-Dose Ketamine (LDK) for post-operative pain, there is growing evidence for using LDK as a clinical alternative to opioids in a palliative care setting. This case study involves monitoring the efficacy of LDK treatment in combination with opioid analgesics in a cancer patient in a hospital setting. This is a very selected case of a patient with Metastatic Prostate Cancer (Gleason 9 Adenocarcinoma) where LDK was shown to be efficacious at reducing pain when opioids and standard pain medications were not satisfactory. While the study involved using a relatively novel pharmacological protocol and close patient monitoring, the patient reported a sustained reduction in pain level based on the Numerical Rating Scale for months after the termination of LDK infusions. Moreover, the treatment also resulted in a reduction of total opioid usage after the addition of LDK. Although additional research is needed to ascertain optimal dosing schedules and route of Ketamine, given these promising findings, Ketamine may be a useful option for improving the treatment of refractory pain in patients with cancer and a good tool in palliative medicine for treating neoplastic pain.
\end{abstract}

Keywords: Low-dose ketamine (LDK); palliative care; neoplastic pain; cancer; case report

Submitted Aug 24, 2020. Accepted for publication Dec 22, 2020.

doi: $10.21037 / \mathrm{apm}-20-1685$

View this article at: http://dx.doi.org/10.21037/apm-20-1685

\section{Introduction}

Pain is the most common symptom for patients diagnosed with cancer; the World Health Organization (WHO) cites that $55-66 \%$ of cancer patients experienced greater than moderate pain despite treatment in a pain management unit (1). While opioids remain to be the mainstay of cancer pain treatment, patients on these medications endure exacerbating side effects and complications. Additionally, due to opioid tolerance, or reduced responsiveness, 10 -fold increases in opioid doses are common in chronic pain management (2). This rapid increase in dosages is problematic due to the narrow therapeutic range of opioids and their high-risk side effects, such as nausea/vomiting, constipation, sedation, drowsiness, and neurological effects such as neuroexcitation, myoclonus, and urinary retention $(3,4)$. The need for non-opioid treatment options for patients undergoing acute, chronic and obstinate pain is of great importance; one alternative is low-dose ketamine (LDK) infusion.

$\wedge$ ORCID: 0000-0002-7521-2951. 
Table 1 Patient total opioid usage (opioid oral MME) averaged (I) 30 days before LDK infusion, (II) during LDK infusion, (III) 30 days after ketamine infusion. Opioid oral MME was calculating by taking the average total daily dose of a drug and multiplying it by a drug-specific conversion factor. The conversion factors that were used are as following: Hydromorphone, IV [20]; Oxycodone, PO [1.5]; Fentanyl, Transdermal Patch [2.4]; Methadone, PO \{1-20 mg [4], 21-40 mg [8], 41-60 mg [10], > $61 \mathrm{mg}$ [12]\}

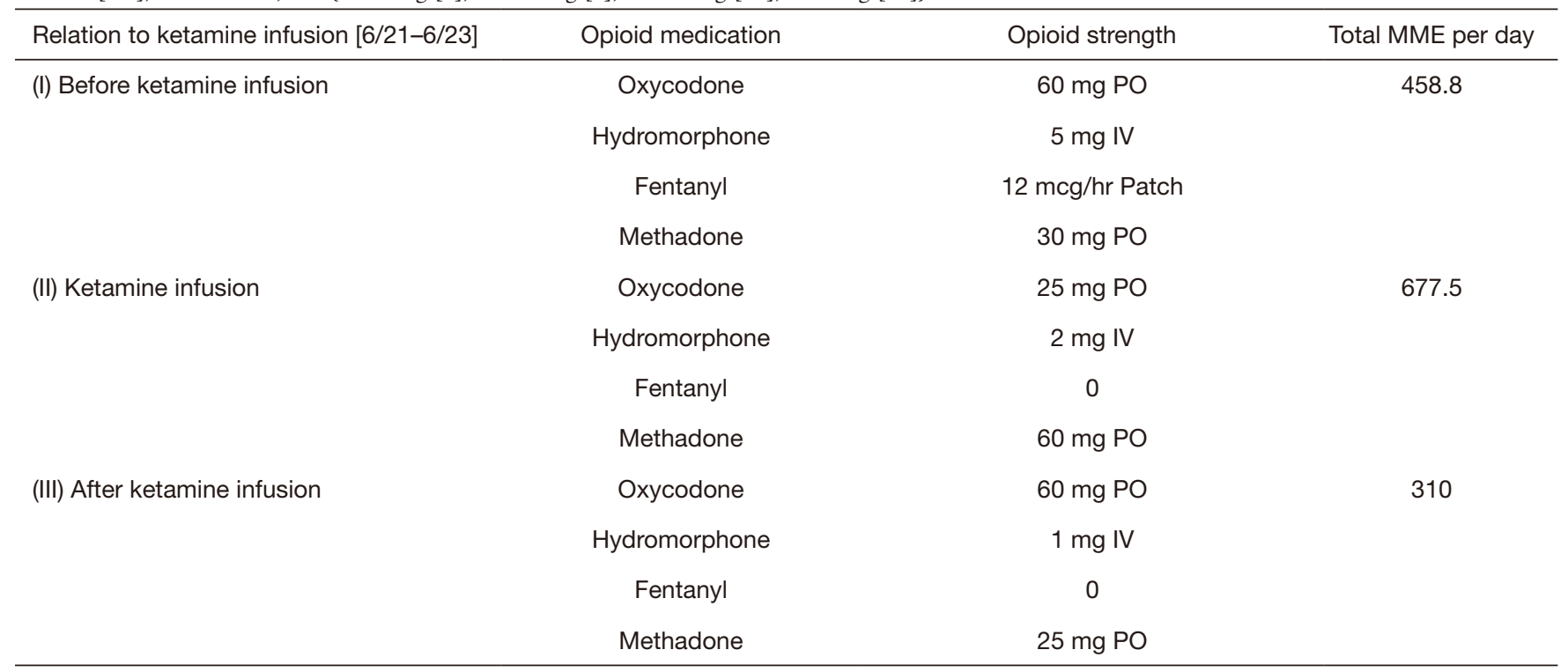

MME, morphine milligram equivalent; LDK, low-dose ketamine.

Here we report a case with Metastatic Prostate Cancer (Gleason 9 Adenocarcinoma), where LDK was used following an initial opioid-based pain management treatment to better manage pain and reduce complications from high-dose opioid use. We present the following article in accordance with the CARE Reporting Checklist (available at http://dx.doi.org/10.21037/apm-20-1685).

\section{Case presentation}

The current case study received approval from the institutional review board. The patient, a 55-year-old male, presented with a 2-year history of Metastatic Prostate Cancer (Gleason 9 Adenocarcinoma) and was referred to the palliative care for secondary unmanageable lower back pain and local hip pain. The etiology of the neuropathic and somatic pain was from bone metastasis that was confirmed with imaging studies. The patient had a history of atrial fibrillation with a rapid ventricular response, dyslipidemia, hypertension, obesity, heart palpitations, sleep apnea, stroke, as well as prostate cancer which metastasized to the bone. The patient was prescribed a myriad of medications including Atorvastatin (Lipitor), Bisacodyl (Dulcolax), Gabapentin, Metoprolol (Toprol XR), as well as several opioid painkillers, such as fentanyl, oxycodone, and hydromorphone. The use of NSAIDs such as Ketorolac was limited due to the potential onset of GI bleeding and acute renal failure. The patient also had recurring issues with constipation, a likely side effect of the opioid medications he was taking. As a result of the opioid-induced constipation, the patient was given a bowel regimen of Senna $8.6 \mathrm{mg}$ PO nightly, Miralax $17 \mathrm{~g}$ POM daily, Bisacodyl suppository and tablet $10 \mathrm{mg}$, and Dexamethasone $6 \mathrm{mg}$ PO daily.

The main criteria used to assess the patient's pain was the Numerical Rating Scale (NRS), which was incorporated into the Physical Exam. The patient consistently reported a 9/10 on the NRS as well as frequent bouts of constipation. An obvious diagnostic challenge faced during the study was the subjective nature of the NRS.

The patient was receiving 458.8 morphine milligram equivalents (MME) per day (Table 1); however, was still reporting an average pain of $9 / 10$ on the NRS. Clinical judgement for the patient was to not drastically increase the patient's opioid usage due to present adverse effects, such as nausea, constipation, urinary retention, lethargy, and ascending drug tolerance. To avoid undesirable effects of high dosage opioids, patient was considered for the LDK infusion. The patient under observation was deemed to have been on the maximum dosages of certain opioids and was at risk of acquiring opioid-induced hyperanalgesia. 
Table 2 Institutional LDK intravenous medication administration guidelines illustrating the proper use of the pharmacotherapy approved for pain management in this patient

\begin{tabular}{|c|c|c|c|c|c|c|}
\hline Medication & \multicolumn{2}{|r|}{ IV push } & \multicolumn{2}{|c|}{ Continuous infusion } & $\begin{array}{l}\text { - Administration } \\
\text { considerations }\end{array}$ & Pharmacy stability \\
\hline
\end{tabular}

LDK, low-dose ketamine.

As a result, LDK infusions were deemed appropriate and potential benefits were clearly observed in this case.

A local LDK infusion protocol has been established in 2019 under federal and institutional guidelines and allowed the use of intravenous LDK titration $(<0.5 \mathrm{mg} / \mathrm{kg})$ as an adjunct to opioid treatment (Table 2). Under these guidelines, the standard concentration of the continuous infusion of Ketamine (Ketalar) was $100 \mathrm{mg}$ in dextrose $5 \% 100 \mathrm{~mL}$ $(1 \mathrm{mg} / \mathrm{mL}$ ) with the maximum push dose being $50 \mathrm{mg} / \mathrm{mL}$ over 1 minute. The 3-day infusion was doses as following: $0.1 \mathrm{mg} / \mathrm{kg} / \mathrm{hr}$ on Day 1 , followed by $0.2 \mathrm{mg} / \mathrm{kg} / \mathrm{hr}$ on Day 2 , followed by $0.3 \mathrm{mg} / \mathrm{kg} / \mathrm{hr}$ on Day 3 (Figure 1) (5-8).

Observational data was collected within the first 24 hours of LDK infusion regarding pain characteristics, pain score assessment, vitals, respiratory depression, tolerance and overall satisfaction. The pain intensity and regular opioid dose before starting and upon stopping Ketamine infusion was also compared for 30 days. Additionally, the NRS was routinely monitored every 4 hours while the patient was on the LDK infusion (Figure 1). Prior to undergoing the LDK infusion, the patient reported a pain level of 9 on the NRS, with 10 being identified as "maximal pain". Despite being in substantial pain, the patient was undergoing Palliative Care treatment of the Equianalgesic Dose of 458.8 MME. After 3 subsequent daily infusions, the patient had experienced a daily alleviation of pain to the level of 5 on the NRS. These results manifested alongside a $32.43 \%$ reduction in opioid usage. The most significant results were evident further after the termination of the Ketamine Infusion. Over a period of 30 days following the Ketamine Infusion, the patient reported an average pain level of 4.75 on the NRS, an approximately $50 \%$ reduction in pain from the start of the Ketamine Infusion. The patient was closely monitored for ketamine side effects, especially within the first hour following each infusion. Common side effects include hallucinations and feelings of agitation; however, the patient did not report any of these adverse effects. On the other hand, the patient reported feeling less total pain following LDK administration. Additionally, opioid usage dropped to 310 MME a $16.67 \%$ drop in Methadone use and a complete termination of Fentanyl use.

Overall, the results obtained point toward the effectiveness of LDK infusions in a Palliative Care setting. These infusions not only alleviated symptoms of pain, but also allowed for a reduction in the use of opioids, and alleviation of possible opioid dependence and associated side effects. No additional adverse events were noted.

All procedures performed in studies involving human participants were in accordance with the ethical standards of the institutional and/or national research committee(s) and with the Helsinki Declaration (as revised in 2013). Due to the fact that the patient under observation had passed away, informed consent was unable to be obtained. Patient's next of kin were untraceable despite the authors' great efforts to obtain their consent.

\section{Discussion}

Onset of acute pain is a significant issue at all points of the cancer continuum and can occur because of malignant conditions or the treatment plan. Earlier studies confirm that more than $50 \%$ of all patients with cancer experience moderate to severe pain (9). The prevalence of pain in cancer survivors is estimated to be $40 \%$, while close to twothirds of those with advanced disease live with pain (10). Cancer pain management, an often-neglected component of oncological care, is closely associated to the development of the modern hospice measures, and palliative care (11). Additionally, the levels of pain experienced are often difficult to assess due to concerns of patients. In a study involving cancer patients $(\mathrm{n}=270)$, difficulty in properly medicating patients was found to be from patient concerns including fear of addiction, reluctancy to complain, and side effects of medications (12). 


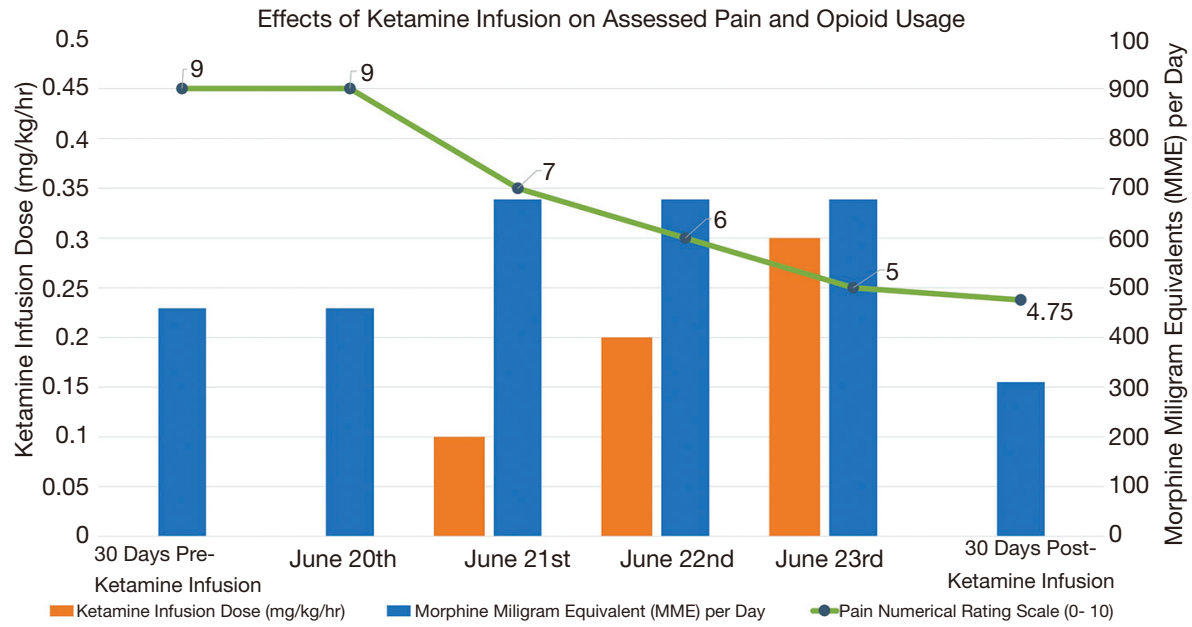

Figure 1 Patient's daily pain numerical rating scale recorded alongside (I) LDK infusion dose and (II) opioid usage. Prior to undergoing LDK infusion, the patient was experiencing consistent levels of high pain, averaging between 8-9 on the Pain Numerical Rating Scale. Upon administration on the LDK infusion, the patient's pain has dropped significantly and averaged between 4-5 on the Pain Numerical Rating Scale for weeks following the treatment. Additionally, the patients need for opioids has decreased dramatically. LDK, low-dose ketamine.

Cancer patients are frequently resorted to opioid use for pain management. These patients may suffer from under-treatment for their pain and/or adverse opioid use outcomes including unnecessary and persistent opioid use $(13,14)$. Moreover, patients with cancer-related pain are underrepresented in the opioid literature despite high opioid exposure (15). While progress has been made in the management of cancer pain, under-treatment persists and does have significant negative impacts on the quality of life of patients (16). The need for non-opioid analgesics for patients undergoing treatment for acute, chronic, and obstinate pain, calls for safer and more efficacious alternatives.

This report illustrates the potential benefits of using ketamine as an adjunct to high dose opioid usage in the palliative care setting. The patient under observation was dealing with metastatic prostate cancer which had spread throughout his pelvic bones. There were multiple fractures seen on CT on $9 / 21 / 2019$, indicating the severity of the metastatic disease. The patient, prior to receiving LDK infusions for a 5-day period, was progressively noted to have dampened spirits due to the disease and poor prognosis. The patient, prior to having cancer, was a very active and enthusiastic individual; the inability to participate in sports such as hiking depressed the patient's mood immensely. A hospital note written by one of the nurses after the LDK infusion mentioned that the patient was in "great spirits", portraying the potential benefit of low-dose ketamine in palliative care settings. Ketamine has been shown to have anti-depressive actions in addition to its ability to relieve pain. An interesting question then arises as to whether Ketamine's actions on NMDA receptors specifically target physical pain, or whether there is an emotional component to its analgesic effect, as well.

Ketamine is a well-known N-methyl-D-Aspartate (NMDA) receptor antagonist, and its analgesic effect is attributed by blocking NMDA receptor induced pain sensitization. Ketamine induces analgesia by prolonging the effect of opioid stimulation by improving the sensitization of opioid receptors (17). There is increasing evidence that LDK has been progressing as a relatively simple, reliable and robust pain reliever in various painful conditions (15-17). The majority of cancer patients with advanced disease have severe pain which significantly impairs health-related quality of life (18). Despite the current emphasis on multimodal analgesic therapy, the potential treatment for pain almost always includes opioids when dealing with advanced stage cancer patients. Given the side effects and dependency issues, there is a strong need of alternate medications. Recent studies have shown that LDK as an adjuvant to opioids significantly reduces the intensity of pain in cancer patients $(19,20)$. The data from these cancer pain control studies exhibit few subjects and involve heterogeneous interventions and outcome measures assessed by different pain assessment tools. Additionally, LDK titrated dose trials are still needed in multiple pain scenarios before it can be declared as a safe analgesic. 


\section{Conclusions}

Cancer pain management, an often neglected component of oncological care, is closely associated to the development of modern hospice measures and palliative care. Cancer patients are frequently resorted to opioid use for pain management, sometimes suffering from under-treatment for their pain and/or adverse opioid use outcomes, including unnecessary and persistent opioid use. This is a very selective case of a patient where LDK infusion was shown to be efficacious at reducing pain when opioids and standard pain medications were not satisfactory. While ketamine use in managing post-operative pain is common, LDK infusion $(<0.5 \mathrm{mg} / \mathrm{kg})$ also shows further potential to deliver excellent outcomes in pain management for cancer patients while simultaneously curbing the myriad of potential side effects of opioids through minimizing their usage. Although additional research is still required to determine the efficacy, optimal dosing schedules, administration routes of LDK infusion, it is hard to underscore the untapped potential of this new treatment in palliative medicine.

\section{Acknowledgments}

Funding: None.

\section{Footnote}

Reporting Checklist: The authors have completed the CARE reporting checklist. Available at http://dx.doi.org/10.21037/ apm-20-1685

Peer Review File: Available at http://dx.doi.org/10.21037/ apm-20-1685

Conflicts of Interest: All authors have completed the ICMJE uniform disclosure form (available at http://dx.doi. org/10.21037/apm-20-1685). The authors have no conflicts of interest to declare.

Ethical Statement: The authors are accountable for all aspects of the work in ensuring that questions related to the accuracy or integrity of any part of the work are appropriately investigated and resolved. All procedures performed in studies involving human participants were in accordance with the ethical standards of the institutional and/or national research committee(s) and with the Helsinki
Declaration (as revised in 2013). Due to the fact that the patient under observation had passed away, informed consent was unable to be obtained. Patient's next of kin were untraceable despite the authors' great efforts to obtain their consent.

Open Access Statement: This is an Open Access article distributed in accordance with the Creative Commons Attribution-NonCommercial-NoDerivs 4.0 International License (CC BY-NC-ND 4.0), which permits the noncommercial replication and distribution of the article with the strict proviso that no changes or edits are made and the original work is properly cited (including links to both the formal publication through the relevant DOI and the license). See: https://creativecommons.org/licenses/by-nc-nd/4.0/.

\section{References}

1. WHO. WHO Guidelines for the Pharmacological and Radiotherapeutic Management of Cancer Pain in Adults and Adolescents. Available online: https://www.who.int/ncds/ management/palliative-care/cancer-pain-guidelines/en/

2. Morgan MM, Christie MJ. Analysis of opioid efficacy, tolerance, addiction and dependence from cell culture to human. Br J Pharmacol 2011;164:1322-34.

3. Caraceni A, Hanks G, Kaasa S, et al. Use of opioid analgesics in the treatment of cancer pain: evidencebased recommendations from the EAPC. Lancet Oncol 2012;13:e58-e68.

4. Benyamin R, Trescot AM, Datta S, et al. Opioid complications and side effects. Pain Physician 2008;11:S105-S120.

5. Ketamine [package insert]. Chestnut Ridge, NY: Par Pharmaceutical; 2017.

6. Groetzinger LM, Rivosecchi RM, Bain W, et al. Ketamine Infusion for Adjunct Sedation in Mechanically Ventilated Adults. Pharmacotherapy 2018;38:181-8.

7. Devlin JW, Skrobik Y, Gélinas C, et al. Clinical Practice Guidelines for the Prevention and Management of Pain, Agitation/Sedation, Delirium, Immobility, and Sleep Disruption in Adult Patients in the ICU. Crit Care Med 2018;46:e825-e873.

8. Erstad BL, Patanwala AE. Ketamine for analgosedation in critically ill patients. J Crit Care 2016;35:145-9.

9. Bennett MI, Eisenberg E, Ahmedzai SH, et al. Standards for the management of cancer-related pain across Europe-A position paper from the EFIC Task Force on Cancer Pain. Eur J Pain 2019;23:660-8. 
10. Pergolizzi J, Böger RH, Budd K, et al. Opioids and the management of chronic severe pain in the elderly: consensus statement of an International Expert Panel with focus on the six clinically most often used World Health Organization Step III opioids (buprenorphine, fentanyl, hydromorphone, methadone, morphine, oxycodone). Pain Pract 2008;8:287-313.

11. Cleary JF. Restoring balance to cancer pain management. Cancer 2020;126:697-700.

12. Ward SE, Goldberg N, Miller-McCauley V, et al. Patientrelated barriers to management of cancer pain. Pain 1993;52:319-24.

13. Binhas M, Krakowski I, Marty J. Nociceptive cancer pain in adult patients: statement about guidelines related to the use of antinociceptive medicine. Ann Fr Anesth Reanim 2007;26:502-15

14. Hattori S, Kimura N, Takatani J, et al. Parenteral opioids in end-of-life care in cancer patients. Gan To Kagaku Ryoho 2005;32:161-6.

15. Carver TW, Kugler NW, Juul J, et al. Ketamine infusion for pain control in adult patients with multiple rib fractures: Results of a randomized control trial. J Trauma Acute Care Surg 2019;86:181-8.

Cite this article as: Patel S, Tatachar V, Singh AB, Galea J, Fattakhov E, Kaur G. Low-dose ketamine as an adjuvant for pain control in a cancer patient: a case report. Ann Palliat Med 2021;10(7):8328-8333. doi: 10.21037/apm-20-1685
16. Walters MK, Farhat J, Bischoff J, et al. Ketamine as an Analgesic Adjuvant in Adult Trauma Intensive Care Unit Patients With Rib Fracture. Ann Pharmacother 2018;52:849-854.

17. Pourmand A, Mazer-Amirshahi M, Royall C, et al. Low dose ketamine use in the emergency department, a new direction in pain management. Am J Emerg Med 2017;35:918-21.

18. Beaudoin FL, Lin C, Guan W, et al. Low-dose ketamine improves pain relief in patients receiving intravenous opioids for acute pain in the emergency department: results of a randomized, double-blind, clinical trial. Acad Emerg Med 2014;21:1193-202.

19. Courade M, Bertrand A, Guerrini-Rousseau L, et al. Low-dose ketamine adjuvant treatment for refractory pain in children, adolescents and young adults with cancer: a pilot study. BMJ Support Palliat Care. 2019;bmjspcare-2018-001739. [Epub ahead of print]. doi:10.1136/bmjspcare-2018-001739.

20. Cheung KWA, Chan PC, Lo SH. The use of ketamine in the management of refractory cancer pain in a palliative care unit. Ann Palliat Med 2020;9:4478-89. 